Shiva Mirkalantari, Faramarz Masjedian, Gholamreza Irajian, Emmanuel Edwar Siddig and Azam Fattahi*

\title{
Determination of the frequency of $\beta$-lactamase genes (bla SHV, bla TEM, bla CTX-M) and phylogenetic groups among ESBL-producing uropathogenic Escherichia coli isolated from outpatients
}

https://doi.org/10.1515/labmed-2018-0136

Received September 13, 2018; accepted September 6, 2019; previously published online November 13, 2019

\section{Abstract}

Background: Escherichia coli accounts for $70-95 \%$ of community-acquired urinary tract infections (UTIs). Recently, there has been an increase in the prevalence of extended-spectrum $\beta$-lactamase (ESBL) in the community which required an accurate identification for better management. Therefore, the current study was performed to determine the antimicrobial resistance pattern, investigate ESBL phenotypes and genotypes (blaCTX-M, bla TEM and bla SHV genes) and determine the phylogenetic groups among ESBL-positive isolates from outpatients.

Methods: One hundred and eighty-three positive urine samples were collected from 4450 outpatient clinic attendees. Antibiotic susceptibility was determined and ESBL phenotype screening was carried out using disk diffusion agar and combination disk techniques, respectively. The assessment of the presence of the blaCTX-M, bla TEM and blaSHV genes and phylogenetic grouping were performed using the polymerase chain reaction (PCR) method.

Results: Out of 183 E. coli isolates, 59 (32.2\%) showed a positive ESBL phenotype. The prevalence of ESBL-producing E. coli was higher in males (57.4\%). Fifty-seven of the ESBL-producing strains carried at least one of the $\beta$-lactamase genes (bla CTX-M, bla TEM, bla SHV). Phylotyping of multi-drug resistant isolates indicated

\footnotetext{
*Correspondence: Azam Fattahi, Department of Medical Parasitology and Mycology, School of Public Health, Tehran University of Medical Sciences, Tehran, Iran,

E-Mail: Afattahi@sina.tums.ac.ir

Shiva Mirkalantari, Faramarz Masjedian and Gholamreza Irajian: Microbiology Department, Faculty of Medicine, Iran University of Medical Sciences, Tehran, Iran

Emmanuel Edwar Siddig: Mycetoma Research Center, University of Khartoum, Khartoum, Sudan
}

that the isolates belonged to B2, A and D phylogroups. Analysis of resistance patterns among these phylogroups revealed that $74.4 \%, 55.3 \%$ and $29.7 \%$ of the isolates in the B2 group were resistant to trimethoprim-sulfamethoxazole, ciprofloxacin and gentamicin, respectively. Most of the strains in the phylogroup B2 carried the bla CTX-M gene.

Conclusions: All the ESBL-producing isolates were placed in one of the four phylogenetic groups. The presence of CTX-M and resistance to quinolones were more frequent in B2 strains than in non-B2 strains.

Keywords: Escherichia coli; extended spectrum $\beta$-lactamase (ESBL); PCR; phylogenetic groups; urinary tract infection.

\section{Introduction}

Urinary tract infections (UTIs) are considered to be one of the most important and frequent infectious diseases, especially among adults. Annually, 150 million UTIs occur around the world [1]. Escherichia coli, from the Enterobacteriaceae family, is the most frequent bacterial agent in both nosocomial and community-acquired UTIs. About $70-80 \%$ of all uropathogens is E. coli [1-3]. UTI is the second most prevalent bacterial infection in the community, and uropathogenic $E$. coli accounts for $70-95 \%$ of community-acquired UTI cases [4-6]. $\beta$-Lactam antibiotics due to their broad antibacterial spectrum and minimal side effects are widely used in the treatment of various infections such as UTI [7]. Interestingly, the non-rational and intensive use of antibiotics by humans and animals is considered as one of the mechanisms that can accelerate the process by which bacteria can acquire resistance [8]. Therefore, the overuse of antibiotics explains the emerging multiple drug resistance in $E$. coli. The resistance of community-acquired $E$. coli to extended spectrum $\beta$-lactamases (ESBLs) is a particular concern for clinicians 
and public health authorities [9]. ESBLs are a group of enzymes that hydrolyze oxyimino- $\beta$-lactams including extended-spectrum cephalosporins but excluding cephamycins and carbapenems [10]. Many different types of ESBLs have been described until now. However, the most common ones are derivatives of the SHV, TEM and CTX-M enzymes [11]. ESBL-producing E. coli isolates are genetically diverse. Recently, the different antibacterial resistance of $E$. coli strains among different phylogenetic groups has been considered as a matter of importance. Phylogenetic analyses have been used for the differentiation of $E$. coli strains into four major phylogenetic groups including A, B1, B2 and D. Three genetic markers (the ChuA, YjaA genes, and the DNA fragment TspE4.c2) have been used as a target in the triplex PCR method to describe phylogenetic groups [12, 13].

It is very important to identify the exact prevalence of ESBL-producing E. coli among Iranian patients with UTI infection for proper patient management. To address this question, as a proof of principle it is vital to determine the antimicrobial resistance patterns, investigate ESBL phenotypes and genotypes (bla CTX-M, bla TEM, bla SHV) and determine the phylogenetic groups among ESBL-positive isolates from outpatient clinics.

\section{Materials and methods}

\section{Study design}

This descriptive, cross-sectional study was conducted during the period from 2014 to 2016 to determine the frequency of phylogenetic groups and $\beta$-lactamase genes (bla CTX-M, bla TEM, bla SHV) of uropathogenic E. coli in outpatients. Eligible subjects received detailed explanations about the study, and informed consent was obtained from all participants.

\section{Subjects and procedure}

The study included 4450 outpatients. First-void urine (FVU) samples were collected in sterile urine containers from these patients, and immediately the samples were inoculated on Eosin methylene blue agar medium and incubated in aerobic condition at $37^{\circ} \mathrm{C}$ for $24-48 \mathrm{~h}$. The counts of $10^{5} \mathrm{cfu} / \mathrm{mL}$ or more was considered as positive. Bacterial identification was made using culture characteristics, Gram stain and biochemical tests.

\section{Antimicrobial susceptibility testing}

Reference susceptibility testing (Kirby-Bauer disk diffusion method) was performed using the available antibiotic disks (Rosco Diagnostica, Taastrup, Denmark) as recommended by the Clinical Laboratory Standard Institute (CLSI) [14]. Antibiotic disks containing cefotaxime $(30 \mu \mathrm{g})$, ceftazidime (30 $\mu \mathrm{g})$, nalidixic acid (30 $\mu \mathrm{g})$, trimethoprime-sulfamethoxazole $(12.5 / 23.75 \mu \mathrm{g})$, amikacin $(30 \mu \mathrm{g})$, ciprofloxacin $(5 \mu \mathrm{g})$, gentamicin $(10 \mu \mathrm{g})$, nitrofrantoin $(30 \mu \mathrm{g})$ and imipenem (10 $\mu \mathrm{g}$ ) were tested against the identified isolates. Escherichia coli ATCC25922 was used as the quality control.

The test was conducted by applying a bacterial inoculum according to the 0.5 McFarland standard on the surface of Mueller-Hinton agar (Merck, Germany) plates. Nine commercially prepared, fixed concentration disks were placed on the inoculated agar. All plates were incubated at $35^{\circ} \mathrm{C}$ for $24 \mathrm{~h}$, and the zones of growth inhibition around each of the antibiotic disks are measured to the nearest millimeter. The zone diameters of each drug are interpreted using the criteria published by the CLSI [14].

\section{Confirmatory test for ESBL detection}

All of the E. coli isolates which were resistant to cefotaxime (30 $\mu \mathrm{g})$ or ceftazidime $(30 \mu \mathrm{g})$ were subjected to ESBL screening according to the CLSI criteria. Both ceftazidime and cefotaxime with and without clavulanic acid $(10 \mu \mathrm{g})$ disks were placed $25 \mathrm{~mm}$ apart from each other on Muller-Hinton agar inoculated with $0.5 \mathrm{McF}$ arland suspension of the test isolate. The difference between the inhibition zone diameters of cefotaxime or ceftazidime disk in combination with clavulanic acid was considered as a positive ESBL phenotype.

\section{Molecular analysis}

\section{DNA extraction}

Genomic DNA was extracted with AccuPrep Genomic DNA Extraction Kit (Bioneer, Korea) and the extraction protocol was followed according to the manufacturer's instructions. The quantity of the extracted DNA was assessed using a spectrophotometer [15]. Then all DNA samples were stored at $-20^{\circ} \mathrm{C}$.

\section{PCR with $\beta$-lactamase-specific primers}

The PCR reaction mixture contained $2 \mu \mathrm{L}$ of extracted DNA, 12.5 $\mu \mathrm{L}$ of Taq DNA Polymerase Master Mix RED 
(Ampliqon, Denmark), $1 \mu \mathrm{L}$ of (bla CTX-M, bla TEM, bla $S H V$ ) primer pairs, $1 \mu \mathrm{L}$ template and $\mathrm{ddH}_{2} \mathrm{O}$ up to a total volume of $25 \mu \mathrm{L}$. The PCR conditions for amplification of all genes were as follows: initial denaturation for $5 \mathrm{~min}$ at $94^{\circ} \mathrm{C}, 35$ cycles of denaturation for $60 \mathrm{~s}$ at $94^{\circ} \mathrm{C}$, annealing for $60 \mathrm{~s}$ at $56^{\circ} \mathrm{C}$ and extension for $90 \mathrm{~s}$ at $72{ }^{\circ} \mathrm{C}$, with a final extension at $72{ }^{\circ} \mathrm{C}$ for $10 \mathrm{~min}$.

\section{Triplex PCR assay}

The phylogenetic group of each ESBL-producing $E$. coli was determined according to Clerment et al. [12] by triplex PCR for the chuA and yjaA genes and the DNA fragment TSPE4.C.

Triplex PCR was performed for the rapid phylogenetic classification of $E$. coli isolates as follows: The reaction mixture included $1 \mu \mathrm{L}$ of extracted DNA, $25 \mu \mathrm{L}$ of Taq DNA Polymerase Master Mix RED (Ampliqon, Denmark), $0.5 \mu \mathrm{L}$ of each specific oligonucleotide primers (Table 1) in a total volume of $50 \mu \mathrm{L}$.

The Triplex PCR cycling parameters were $95{ }^{\circ} \mathrm{C}$ for $10 \mathrm{~min}, 35$ cycles of denaturation for $40 \mathrm{~s}$ at $95^{\circ} \mathrm{C}$, annealing for $40 \mathrm{~s}$ at $57^{\circ} \mathrm{C}$, an extension for $40 \mathrm{~s}$ at $72^{\circ} \mathrm{C}$, with a final extension of $8 \mathrm{~min}$ at $72{ }^{\circ} \mathrm{C}$. Escherichia coli ATCC25922 was used as a control strain for each cycle.

\section{Gel electrophoresis}

All PCR products were visualized by $1.5 \%$ agarose gel electrophoresis in Tris-borate-ethylenediaminetetraacetic acid (TBE) buffer and stained with CinnaGen DNA Safe Stain (SinaClon BioScience Co., Iran).

Table 1: Oligonucleotides used in this study.

\begin{tabular}{|c|c|c|c|}
\hline Primer & Nucleotide sequence $\left(5^{\prime} \rightarrow 3^{\prime}\right)$ & Product size & Ref \\
\hline \multirow[t]{2}{*}{ bla $_{\text {TEM }}$} & F-GAGACAATAACCCTGGTAAAT & $459 \mathrm{bp}$ & [9] \\
\hline & R-AGAAGTAAGTTGGCAGCAGTG & & \\
\hline \multirow{2}{*}{ bla ${ }_{C T X-M}$} & F-GAAGGTCATCAAGAAGGTGCG & $560 \mathrm{bp}$ & [9] \\
\hline & R-GCATTGCCACGCTTTTCATAG & & \\
\hline \multirow[t]{2}{*}{ bla $_{S H V}$} & F-AAGATCCACTATCGCCAGCAG & $231 \mathrm{bp}$ & [15] \\
\hline & R-ATTCAGTTCCGTTTCCCAGCGG & & \\
\hline \multirow[t]{2}{*}{ chuA.1 } & F-GACGAACCAACGGTCAGGAT & 279 & [11] \\
\hline & R-TGCCGCCAGTACCAAAGACA & & \\
\hline \multirow[t]{2}{*}{ yjaA.1 } & F-TGAAGTGTCAGGAGACGCTG & 211 & [11] \\
\hline & R-ATGGAGAATGCGTTCCTCAAC & & \\
\hline \multirow[t]{2}{*}{ TspE4c2.1tgbv } & F-GAGTAATGTCGGGGCATTCA & 152 & [11] \\
\hline & R-CGCGCCAACAAAGTATTACG & & \\
\hline
\end{tabular}

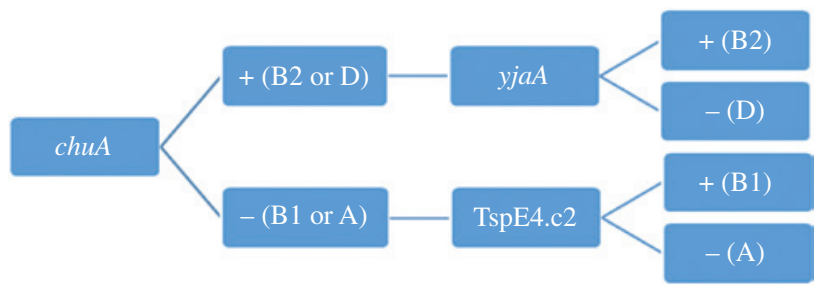

Figure 1: Assigning of different phylogenetic groups.

\section{Designation of different phylogenetic groups}

After electrophoresis, the strains were assigned to the phylogenetic groups as shown in Figure 1.

\section{Ethical considerations}

The study was performed according to the Helsinki principles of ethics. All participants were aware of the study purposes and signed a written informed consent.

\section{Data analysis}

Statistical analyses were performed using the Statistical Package for Social Sciences (SPSS) for Windows 16.0 (SPSS Inc., Chicago, IL, USA).

\section{Results}

Urine samples that met the inclusion criteria of $10^{5}$ colonyforming units per milliliter were subjected to this study. A total of 183 non-duplicate E. coli isolates were recovered from the urine specimens. The mean and standard deviation age of the source patients was $35.3 \pm 22.35$ years (range from 12 months to 90 years). The prevalence of UTI was $159(86.88 \%)$ and $24(13.11 \%)$ in females and males, respectively. There was a significant difference in UTI prevalence between the two sexes $(p<0.05)$.

\section{Antimicrobial susceptibility testing}

For the total isolates the in vitro susceptibilities to cefotaxime, ceftazidime, nalidixic acid, trimethoprime-sulfamethoxazole, amikacin, ciprofloxacin, gentamicin, nitrofrantoin and imipenem were determined by using the disk diffusion assays; our present results showed that 


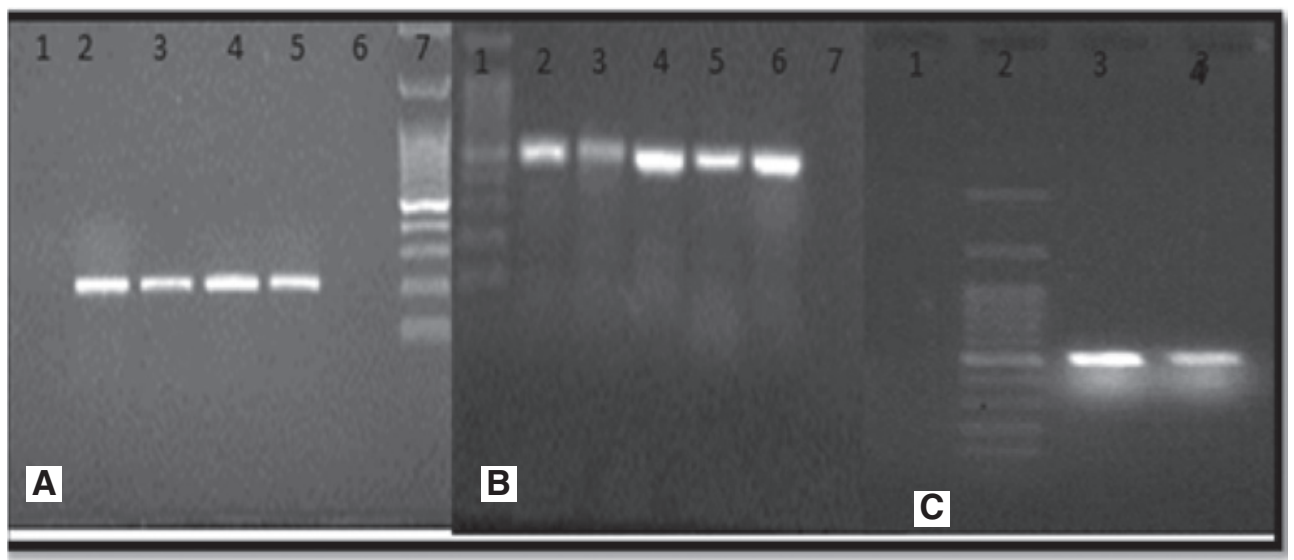

Figure 2: Gel electrophoresis of bla CTX-M, bla TEM, bla SHV PCR products.

Lanes A7, B1, C2 contain a 100 bp DNA ladder, lanes A2 to A5 show PCR products for four samples which were positive for the blaSHV gene (231 bp), lane A6 was a negative control. Lanes B2 to B6 show PCR products for five samples which were positive for blaTEM (459 bp). Lanes C3 and C4 show PCR results for two samples which were positive for the blaCTX-M gene (560 bp).

$1.63 \%$ of isolates were resistant to all antibiotics and $27.3 \%$ of isolates were susceptible to all antibiotics.

Out of 183 E. coli isolates, 59 (32.2\%) showed a positive ESBL phenotype using the Double Disc Synergy Test (DDST) according to CLSI 2014 [14]. ESBL-producing E. coli was higher in males (57.4\%) compared to females (42.6\%). Fifty-seven (96.6\%) of the ESBL-producing E. coli strains carried at least one of the $\beta$-lactamase genes (bla CTX-M, bla TEM, bla SHV).

Interestingly, two (3.4\%) of the phenotypically positive ESBL strains were negative for the (bla CTX-M, bla TEM, bla $S H V$ ) genes.

Furthermore, the results obtained from the current study show a significant association between ESBLproducing $E$. coli and resistance to CAZ $(\mathrm{p}<0.001)$, CTX $(p<0.001)$, trimethoprim-sulfamethoxazole $(p<0.001)$ and ciprofloxacin $(\mathrm{p}<0.05)$.

Fifty percent of the ESBL isolates were found to be sensitive to gentamicin. Resistance to trimethoprimesulfamethoxazole (64.4\%), amikacin (59.3\%) and ciprofloxacin (59.3\%) was observed among the ESBLproducing isolates. All the isolates were found to be highly susceptible to imipenem, nitrofurantoin and nalidixic acid.

\section{Detection of $\beta$-lactamase genes}

Detection of $\beta$-lactamase genes in 59 ESBL-producing $E$. coli isolates showed the presence of $69.5 \%, 47.4 \%$ and $44 \%$ of bla CTX-M, bla TEM, bla SHV genes, respectively, using specific PCR primers (Figure 2). The simultaneous
Table 2: Distribution of different $\beta$-lactamase gene patterns in four phylogroups (A, B1, B2, D).

\begin{tabular}{lrrrrr}
\hline $\begin{array}{l}\text { B-Lactamase } \\
\text { gene patterns }\end{array}$ & n & A B1 & B2 & D \\
\hline bla $_{\text {CTX-M-TEM-SHV }}$ & $19(33.3 \%)$ & - & - & $13(68.4 \%)$ & $6(31.6 \%)$ \\
bla $_{\text {CTX-M-SHV }}$ & $9(15.78 \%)$ & - & - & $9(100 \%)$ & - \\
bla $_{\text {CTX-M-TEM }}$ & $2(3.5 \%)$ & - & - & $2(100 \%)$ & - \\
bla $_{\text {TEM-SHV }}$ & $4(7 \%)$ & $4(100 \%)$ & - & - & - \\
bla $_{\text {TEM }}$ & $4(7 \%)$ & - & - & $4(100 \%)$ & - \\
bla $_{\text {SHV }}$ & $3(5.26 \%)$ & - & - & $3(100 \%)$ & - \\
bla $_{\text {CTX-M }}$ & $16(28 \%)$ & - & - & $16(100 \%)$ & - \\
\hline
\end{tabular}

presence of two (blaCTX-M-SHV or blaCTX-M-TEM or blaTEM-SHV) and three ESBL genes (CTX-TEM-SHV) in a single isolate occurred in $25.4 \%$ and $32.2 \%$ isolates, respectively (Table 2).

Triplex PCR assays for phylotyping of multi-drug resistant isolates indicated that the isolates belonged to the B2, D and A phylogroups (Figure 3). ESBL-positive isolates were found to be more in the phylogenetic group B2 and less in groups A and D. In this study, no strain was found belonging to group B1.

Analysis of the resistance pattern among these phylogroups revealed that $74.4 \%, 55.3 \%$ and $29.7 \%$ of isolates in group B2 were resistant to SXT, CP and GM, respectively. Whereas most of the strains in phylogroup B2 carried the bla CTX-M gene, this result reveals that the bla CTX-M gene simultaneously occurs with other genes resistant to fluoroquinolone and aminoglycoside antibiotics (Table 2). 


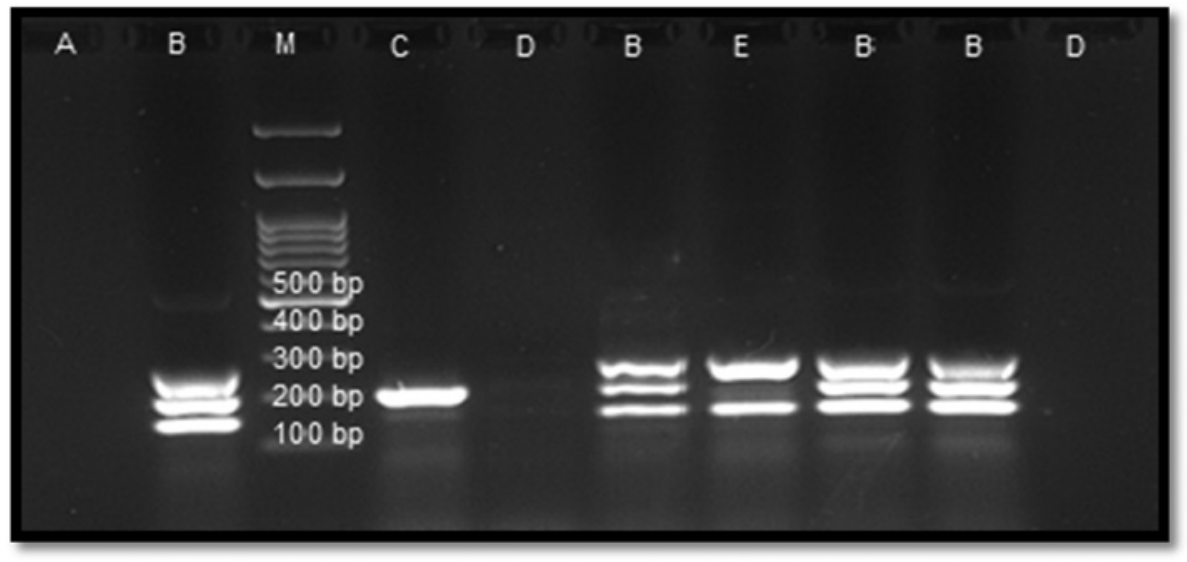

Figure 3: Triplex PCR for phylogenetic groups.

Lane A, negative control; lane B, group B2; lane C, group A; lane D, group A; lane E, group D; M, 100 bp ladder.

\section{Discussion}

Clinical management of UTI is one of the most important aims, because $E$. coli strains are responsible for a high percentage of this infection which can acquire resistance to antimicrobial agents [16-18]. Several antibiotics can be used for the treatment of bacterial infections; however most general practitioners prescribe $\beta$-lactam antibiotics to be used as the first line of UTI treatment. Furthermore, due to uncontrolled consumption of antibiotics nowadays several bacteria have started developing resistance to this class of antimicrobial agents by ESBL enzymes as a mechanism of resistance.

The results of the present study showed that community-acquired UTI is more common in females than in males and the reported results are in agreement with previous studies $[19,20]$. Despite the higher prevalence of UTI among the female population, we demonstrated in this report that 59 of our isolates carried the resistance gene from which $57.4 \%$ were isolated from males. The results of our current report is in concordance with that conducted by Shashwati and his associates, who studied the prevalence of ESBLs among the patients they attended at the tertiary care center; their results showed that ESBL producers were more in males $(52.54 \%)$ than in females [21]. Findings of the present study and previous ones confirmed that male gender is one of the risk factors associated with community-acquired ESBL-positive UTI [22, 23].

Furthermore, we demonstrated in this study that ESBLproducing isolates were resistant to other $\beta$-lactamase antibiotics including trimethoprim-sulfamethoxazole and ciprofloxacin. However, none of the isolates were found to be resistant to imipenem which is considered an effective drug for UTI treatment in many countries [24, 25],
Carbapenems, including imipenem, should be kept in reserve for use in life-threatening infections [21]. Moreover; the results of our current report showed that more than $90 \%$ of our ESBL isolates were susceptible to nitrofurantoin and nalidixic acid.

The result of genotype analyses in the present study showed that $E$. coli isolates carried different types of ESBL genes. Two important risk factors that increase infection by ESBL-producing isolates are the presence of more underlying diseases and higher antibiotic pressure. These risk factors usually exist in elderly patients [26].

The prevalence of $\beta$-lactamase genes is variable across countries, cities and regions. In the current study, the prevalence of bla CTX-M, bla TEM, bla SHV was $44 \%$, 47.4 and $69.5 \%$, respectively $[27,28]$. At the first cases of ESBLs, the prevalence of the TEM and SHV genes was the most predominant but now more CTX-M type is reported from several countries including Africa, India, Iran and industrialized countries such as France, Canada and the UK [29]. In the present study, the predominant ESBL gene in E. coli isolates was CTX-M and two isolates of positive phenotype ESBL isolates lacked CTX-M, SHV and TEM, which can be explained by the possible presence of other ESBL genes. In several similar studies [30, 31], the coexistence of different $\beta$-lactamase genes within the same isolates have been analyzed as well. The results showed that $25.4 \%$ and $32.2 \%$ of the ESBL-producing isolates carry two or three $\beta$-lactamase genes, respectively. Data analyses revealed that CTX-M is the most prevalent ESBL; this finding is consistent with previous studies [31-34]. Production of CTX-M was significantly associated with resistance to most of the antibiotics.

All of the ESBL-producing isolates were placed in one of the four phylogenetic groups A, B1, B2 and D. Among 
E. coli strains, B2 and D phylogenetic groups were the most prevalent in this study. This result was in accordance with other studies $[35,36]$. The results of two independent studies conducted by Saeed and Dadie indicated that most of the isolates belonged to group $\mathrm{A}$, which is in contrast to our study $[37,38]$. This difference in the phylogroup distribution can be due to several factors such as genetic, dietary, health, environmental and geographic conditions of the host [39-41].

According to the present findings, the presence of CTX-M and resistance to quinolones was more frequently observed in group B2 strains than in non-B2 strains which can be explained by simultaneous occurrence of fluoroquinolone resistance genes with the blaCTX-M gene which was observed in several studies such as in Ruppe, Juan and Mosquito [42-44].

In conclusion, uncontrolled consumption of antibiotics in the community has resulted in a high prevalence of ESBL-producing E. coli isolates among community-acquired urinary infections. $\beta$-Lactamase genes particularly CTX-M, TEM and SHV have the capacity to disseminate and persist in the community. In this project, all the ESBL-producing isolates were placed in one of the four phylogenetic groups. The presence of CTX-M and resistance to quinolones was more frequently observed in group B2 strains than in non-B2 strains. In addition, several studies are needed in various regions with a higher population for understanding the distribution of ESBL-producing $E$. coli in the community and for providing phylogenetic information.

Author contributions: All the authors have accepted responsibility for the entire content of this submitted manuscript and approved submission.

Research funding: None declared.

Employment or leadership: None declared.

Honorarium: None declared.

Competing interests: The funding organization(s) played no role in the study design; in the collection, analysis, and interpretation of data; in the writing of the report; or in the decision to submit the report for publication.

\section{References}

1. Mukherjee M, Basu S, Mukherjee SK, Majumder M. Multidrugresistance and extended spectrum beta-lactamase production in uropathogenic $\mathrm{E}$. coli which were isolated from hospitalized patients in Kolkata, India. J Clin Diagn Res 2013;7:449-53.

2. Nickel JC. Urinary Tract Infections and Resistant Bacteria: Highlights of a Symposium at the Combined Meeting of the 25th
International Congress of Chemotherapy (ICC) and the 17th European Congress of Clinical Microbiology and Infectious Diseases (ECCMID), March 31-April 3, 2007, Munich, Germany. Rev Urol 2007;9:78-80.

3. Auer S, Wojna A, Hell M. Oral treatment options for ambulatory patients with urinary tract infections caused by extended-spectrum-beta-lactamase-producing Escherichia coli. Antimicrob Agents Chemother 2010;54:4006-8.

4. Jacobsen SM, Stickler DJ, Mobley HL, Shirtliff ME. Complicated catheter-associated urinary tract infections due to Escherichia coli and Proteus mirabilis. Clin Microbiol Rev 2008;21:26-59.

5. Foxman B. The epidemiology of urinary tract infection. Nat Rev Urol 2010;7:653-60.

6. Rodríguez-Baño J, Alcalá JC, Cisneros JM, Grill F, Oliver A, Horcajada JP, et al. Community infections caused by extendedspectrum $\beta$-lactamase-producing Escherichia coli. Arch Intern Med 2008;168:1897-902.

7. Miao Z, Li S, Wang L, Song W, Zhou Y. Antimicrobial resistance and molecular epidemiology of ESBL-producing Escherichia coli isolated from outpatients in town hospitals of Shandong Province, China. Front Microbiol 2017;8:63.

8. Arul Prakasam KC, Dileesh Kumar KG, Vijayan M. A cross sectional study on distribution of urinary tract infection and their antibiotic utilisation pattern in Kerala. Int J PharmTech Res 2012;4:1309-16.

9. Nicolle LE. Antimicrobial resistance in community-acquired Escherichia coli isolated from urinary infection: Good news or bad? Can J Infect Dis Med Microbiol 2013;24:123-4.

10. Maharjan A, Bhetwal A, Shakya S, Satyal D, Shah S, Joshi G, et al. Ugly bugs in healthy guts! Carriage of multidrug-resistant and ESBL-producing commensal Enterobacteriaceae in the intestine of healthy Nepalese adults. Infect Drug Resist 2018;11:547-54.

11. Arpin C, Quentin C, Grobost F, Cambau E, Robert J, Dubois V, et al. Nationwide survey of extended-spectrum \{beta\}-lactamase-producing Enterobacteriaceae in the French community setting. J Antimicrob Chemother 2009;63:1205-14.

12. Clermont $\mathrm{O}$, Bonacorsi S, Bingen E. Rapid and simple determination of the Escherichia coli phylogenetic group. Appl Environ Microbiol 2000;66:4555-8.

13. Das P, Singh AK, Mukherjee S, Rajendran K, Saha DR, Koley H, et al. Composition of Escherichia coli population in the neonatal gut: phylogroups and virulence determinants. J Med Microbiol 2013;62:1680-7.

14. Wayne P. Clinical and Laboratory Standards Institute: performance standards for antimicrobial susceptibility testing: 20th informational supplement. CLSI document M100-S20, 2010.

15. Muzaheed, Doi Y, Adams-Haduch JM, Shivannavar CT, Paterson DL, Gaddad SM. Faecal carriage of CTX-M-15-producing Klebsiella pneumoniae in patients with acute gastroenteritis. Indian J Med Res 2009;129:599-602.

16. Manges AR, Johnson JR, Foxman B, O'bryan TT, Fullerton KE, Riley LW. Widespread distribution of urinary tract infections caused by a multidrug-resistant Escherichia coli clonal group. $\mathrm{N}$ Engl J Med 2001;345:1007-13.

17. Johnson JR, Kuskowski MA, Owens K, Gajewski A, Winokur PL. Phylogenetic origin and virulence genotype in relation to resistance to fluoroquinolones and/or extended-spectrum cephalosporins and cephamycins among Escherichia coli isolates from animals and humans. J Infect Dis 2003;188:759-68. 
18. Tabar MM, Mirkalantari S, Amoli RI. Detection of ctx-M gene in ESBL-producing E. coli strains isolated from urinary tract infection in Semnan, Iran. Electron Physician 2016;8:2686-90.

19. Prais D, Straussberg R, Avitzur Y, Nussinovitch M, Harel L, Amir J. Bacterial susceptibility to oral antibiotics in community acquired urinary tract infection. Arch Dis Child 2003;88:215-8.

20. Dias Neto JA, Martins AC, Silva LD, Tiraboschi RB, Domingos AL, Cologna AJ, et al. Community acquired urinary tract infection: etiology and bacterial susceptibility. Acta Bras Cir 2003;18:33-6.

21. Shashwati N, Kiran T, Dhanvijay AG. Study of extended spectrum beta-lactamase producing Enterobacteriaceae and antibiotic coresistance in a tertiary care teaching hospital. J Nat Sci Biol Med 2014;5:30-5.

22. Ghazvini H, Taheri K, Edalati E, Sedighi M, Mirkalantari S. Virulence factors and antimicrobial resistance in uropathogenic Escherichia coli strains isolated from cystitis and pyelonephritis. Turk J Med Sci 2019;49:361-7.

23. Shah AA, Hasan F, Ahmed S, Hameed A. Extended-spectrum beta-lactamases in Enterobacteriaceae: related to age and gender. New Microbiol 2002;25:363-6.

24. Akram M, Shahid M, Khan AU. Etiology and antibiotic resistance patterns of community-acquired urinary tract infections in JNMC Hospital Aligarh, India. Ann Clin Microbiol Antimicrob 2007;6:4.

25. Babypadmini S, Appalaraju B. Extended spectrum-lactamases in urinary isolates of Escherichia coli and Klebsiella pneumoniaeprevalence and susceptibility pattern in a tertiary care hospital. Indian J Med Microbiol 2004;22:172-4.

26. Blom A, Ahl J, Mansson F, Resman F, Tham J. The prevalence of ESBL-producing Enterobacteriaceae in a nursing home setting compared with elderly living at home: a cross-sectional comparison. BMC Infect Dis 2016;16:111.

27. Al-Jasser AM. Extended-spectrum beta-lactamases (ESBLs): a global problem. Kuwait Med J 2006;38:171-85.

28. Pokhrel RH, Thapa B, Kafle R, Shah PK, Tribuddharat C. Co-existence of beta-lactamases in clinical isolates of Escherichia coli from Kathmandu, Nepal. BMC Res Notes 2014;7:694.

29. Vranic SM, Uzunovic A. Antimicrobial resistance of escherichia coli strains isolated from urine at outpatient population: a single laboratory experience. Mater Sociomed 2016;28:121-4.

30. Oteo J, Perez-Vazquez M, Campos J. Extended-spectrum [beta]lactamase producing Escherichia coli: changing epidemiology and clinical impact. Curr Opin Infect Dis 2010;23:320-6.

31. Kang Cl, Wi YM, Lee MY, Ko KS, Chung DR, Peck KR, et al. Epidemiology and risk factors of community onset infections caused by extended-spectrum beta-lactamase-producing Escherichia coli strains. J Clin Microbiol 2012;50:312-7.

32. Ben-Ami R, Rodriguez-Bano J, Arslan H, Pitout JD, Quentin C, Calbo ES, et al. A multinational survey of risk factors for infection with extended-spectrum beta-lactamase-producing Enterobacteriaceae in nonhospitalized patients. Clin Infect Dis 2009;49:682-90.
33. Kang Cl, Song JH, Chung DR, Peck KR, Ko KS, Yeom JS, et al. Risk factors and treatment outcomes of community-onset bacteraemia caused by extended-spectrum beta-lactamaseproducing Escherichia coli. Int J Antimicrob Agents 2010;36:284-7.

34. Koljalg S, Truusalu K, Stsepetova J, Pai K, Vainumae I, Sepp E, et al. The Escherichia coli phylogenetic group B2 with integrons prevails in childhood recurrent urinary tract infections. APMIS 2014;122:452-8.

35. Bashir S, Haque A, Sarwar Y, Ali A, Anwar MI. Virulence profile of different phylogenetic groups of locally isolated community acquired uropathogenic E. coli from Faisalabad region of Pakistan. Ann Clin Microbiol Antimicrob 2012;11:23.

36. Luo Y, Ma Y, Zhao Q, Wang L, Guo L, Ye L, et al. Similarity and divergence of phylogenies, antimicrobial susceptibilities, and virulence factor profiles of Escherichia coli isolates causing recurrent urinary tract infections that persist or result from reinfection. J Clin Microbiol 2012;50:4002-7.

37. Saeed MA, Haque A, Ali A, Mohsin M, Bashir S, Tariq A, et al. Relationship of drug resistance to phylogenetic groups of E. coli isolates from wound infections. J Infect Dev Ctries 2009;3:667-70.

38. Dadie A, Kouassi N, Dako E, Dje M, Dosso M. Virulence, serotype and phylogenetic groups of diarrhoeagenic Escherichia coli isolated during digestive infections in Abidjan, Côte d'Ivoire. Afr J Biotechnol 2014;13:998-1008.

39. Duriez P, Clermont O, Bonacorsi S, Bingen E, Chaventre A, Elion J, et al. Commensal Escherichia coli isolates are phylogenetically distributed among geographically distinct human populations. Microbiology 2001;147:1671-6.

40. Mirkalantari S, Jazayeri Moghadas A. Diversity determination of CTX-M1 producing Klebsiella pneumoniae using multilocus variable-number tandem repeat analysis, Semnan, Iran, Jundishapur. J Microbiol 2018;11:e63131.

41. Jazayeri Moghadas A, Kalantari F, Sarfi M, Shahhoseini S, Mirkalantari S. Evaluation of virulence factors and antibiotic resistance patterns in clinical urine isolates of Klebsiella pneumoniae in Semnan, Iran. Jundishapur J Microbiol 2018;11:e63637.

42. Juan C, Horcajada I, Soto S, Gajewski A, Smithson A, Teresa A, et al. Quinolone-resistant uropathogenic Escherichia coli strains from phylogenetic group B2 have fewer virulence factors than their susceptible. J Clin Microbiol 2005;43:2962-4.

43. Mosquito S, Pons MJ, Riveros M, Ruiz J, Ochoa TJ. Diarrheagenic Escherichia coli phylogroups are associated with antibiotic resistance and duration of diarrheal episode. ScientificWorldJournal 2015;2015:6.

44. Ruppé E, Hem S, Lath S, Gautier V, Ariey F, Sarthou L, et al. CTX-M $\beta$-lactamases in Escherichia coli from communityacquired urinary tract infections, Cambodia. Emerg Infect Dis 2009;15:741-8. 